\title{
Geofluid Academy
}

Geofluid Academy

Bonizzella Brizzolari - Associazione Acque Sotterranee, Pisa - b.brizzolari@acquesotterranee.com

Keywords: Geofluid, Geofluid Academy, Associazione Acque Sotterranee, Associazione Acque Sotterranee Scuola e Formazione.

Parole chiave: Geofluid, Geofluid Academy, Associazione Acque Sotterranee, Associazione Acque Sotterranee Scuola e Formazione.

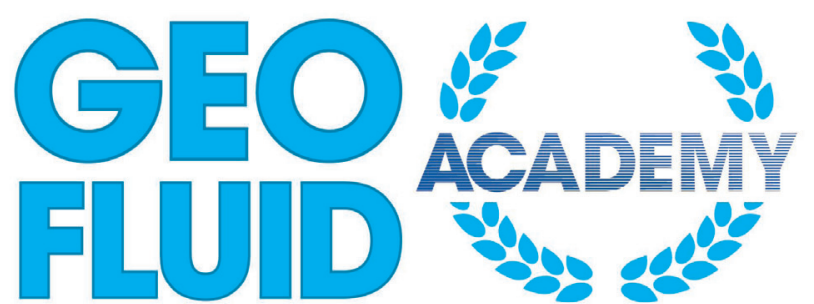

Piacenza Expo propone una nuova iniziativa: Geofluid Academy. Il progetto nasce dalla collaborazione tra Piacenza Expo, ANIPA e Associazione Acque Sotterranee Scuola e Formazione con un duplice obbiettivo:

- $\quad$ arricchire di maggiore specializzazione il successo del GEOFLUID;

- dare ai professionisti del settore un'esperienza "sul campo".

La grande novità per questo settore è proprio quella di proporre una giornata di aggiornamento professionale attraverso una parte teorica svolta in aula e una parte pratica svolta all'esterno del Centro Congressi Piacenza Expo (Fig. 1).

Per il futuro, si immaginano collaborazioni con le altre Associazioni ed Enti per coprire l'intera gamma di interessi dei settori presenti alla manifestazione che avrà cadenza biennale.

Le iniziative di Geofluid Academy verranno, infatti, proposte negli anni dispari, con uno specifico momento formativo ed informativo dedicato di volta in volta a distinte tematiche.

Scopo di Geofluid Academy sarà quello di:

- contribuire alla diffusione delle conoscenze tecnico-scientifiche mediante congressi, convegni, seminari, conferenze, short course e giornate di studio;

- $\quad$ promuovere il confronto tra le imprese, i professionisti e la pubblica amministrazione per una reciproca informazione;

- sviluppare l'approfondimento di temi innovativi per una gestione sostenibile delle risorse e per la tutela dell'ambiente;

- collaborare con le imprese del settore, italiane e straniere, alla formazione del personale.

Il progetto, per il momento, prevede di fornire un supporto teorico-pratico a carattere formativo e divulgativo agli operatori dei seguenti settori:
- $\quad$ ricerca, estrazione e trasporto dei fluidi sotterranei;

- indagini geognostiche e geotecniche, fondazioni speciali e perforazioni direzionali;

- costruzione di gallerie;

- $\quad$ analisi, monitoraggio ambientale, bonifica dei terreni, difesa del suolo e idrogeologia in generale.

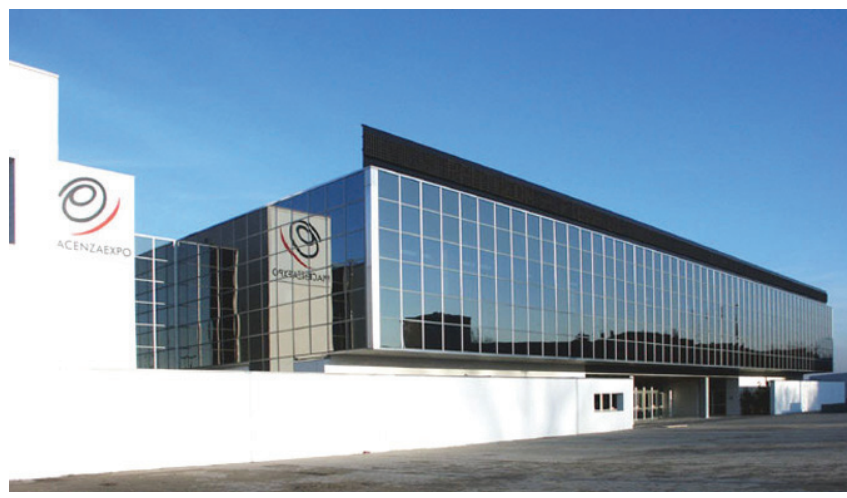

Fig. 1 - Sede di Piacenza Expo.

Fig. 1 - Piacenza Expo Area.

Il primo corso di GEOFLUID ACADEMY si terrà il giorno 8 NOVEMBRE 2019 presso Piacenza Expo.

Si tratterà di una manifestazione formativa - divulgativa che prevede una parte in aula e una parte on site nell'area espositiva esterna del quartiere fieristico.

Qui di seguito il programma provvisorio.

\section{TEMI:}

- Gli impianti di perforazione: caratteristiche principali di identificazione in funzione del o dei sistema/i adottato/i. Questi temi saranno sviluppati in 3 step: prima in aula, poi nel piazzale, poi ancora in aula;

- I principali accessori necessari agli impianti per svolgere la loro funzione: caratteristiche principali di identificazione in funzione del o dei sistema/i adottato/i. 2 step: prima in aula, poi nel piazzale;

- Valutazione delle caratteristiche tecniche al fine di determinare le capacità operative degli impianti e dei relativi accessori. 1 step: in aula;

- Operazioni pratiche da poter svolgere nel piazzale della Fiera. 
Luogo di svolgimento: Piacenza presso il Centro Congressi di Piacenza Expo e la relativa area espositiva esterna.

Durata: dalle 9,30 alle 17,30 del giorno 8 novembre 2019.

A chi è rivolto: geologi e ingegneri che potranno acquisire crediti formativi.

Chi espone: ditte costruttrici di macchinari e/o accessori.

Come si espone: con un macchinario per la prova su campo e/o con un desk nella galleria del centro congressi.

Chi relaziona: tecnici e sviluppatori di macchinari e/o accessori, mondo accademico.
Ulteriori informazioni sono disponibili nel sito di Geofluid (www.geofluid.it) nella sezione Geofluid Academy.

Inoltre chi volesse diventare partner tecnico deve rivolgersi alla dr.ssa Alessandra Bottani (telefono 0523 602711) o inviarle una mail alessandra.bottani@piacenzaexpo.it

Le iscrizioni saranno aperte nel mese di settembre, in data da definire, sul sito www.acquesotterranee.it nella sezione Scuola e Formazione. Il corso sarà a pagamento.

Per informazioni sulle iscrizioni contattare la dr.ssa Bonizzella Brizzolari al 3391501511 o inviarle una mail: b.brizzolari@acquesotterranee.com.

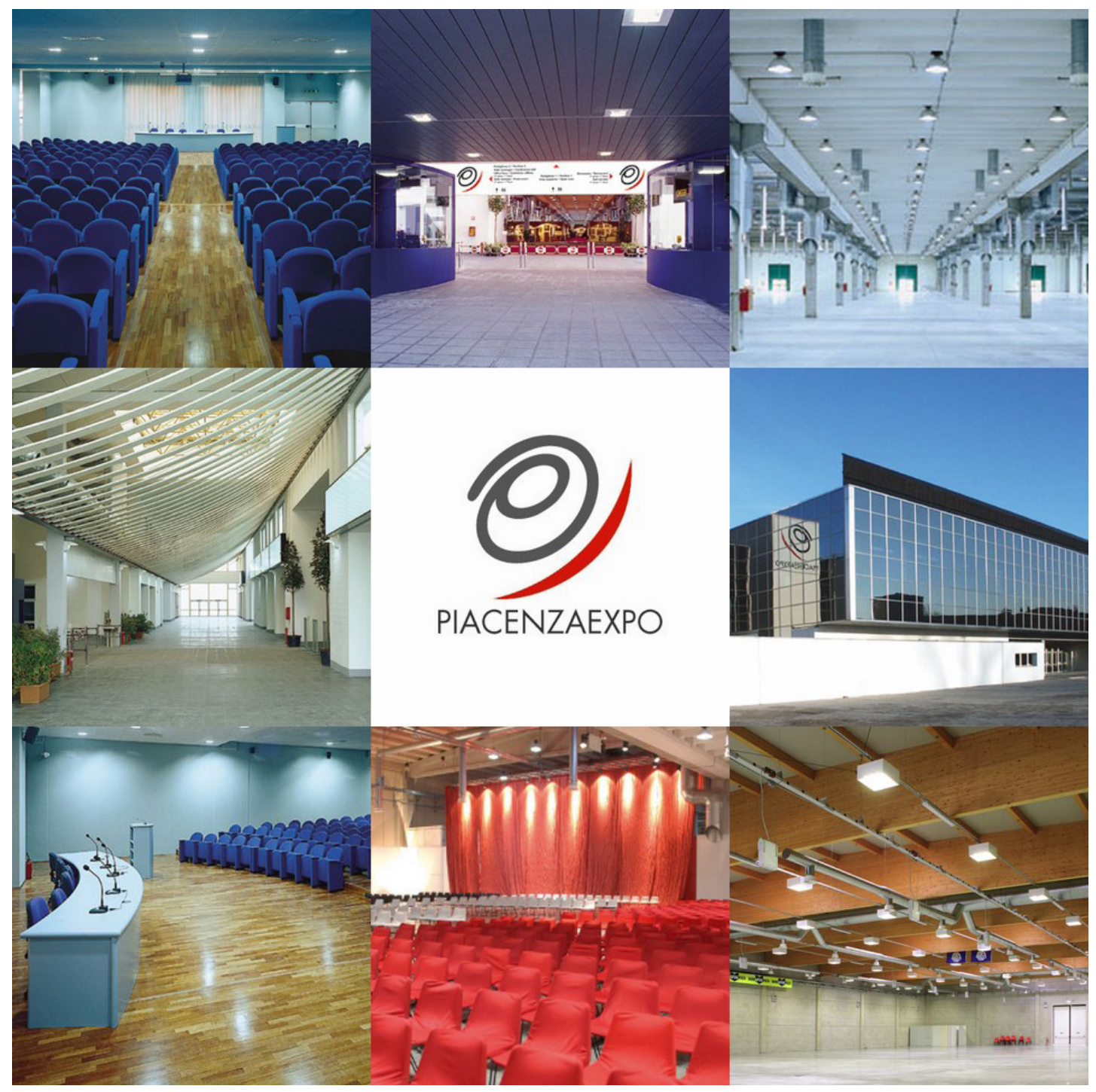

